\title{
Bloch-mode extraction from near-field data in periodic waveguides
}

\author{
Sangwoo Ha, ${ }^{1}$ Andrey A. Sukhorukov, ${ }^{1, *}$ Kokou B. Dossou, ${ }^{2}$ \\ Lindsay C. Botten, ${ }^{2}$ C. Martijn de Sterke, ${ }^{3}$ and Yuri S. Kivshar ${ }^{1}$ \\ ${ }^{1}$ Centre for Ultra-high Bandwidth Devices for Optical Systems (CUDOS), Nonlinear Physics Centre, Research School \\ of Physics and Engineering, Australian National University, Canberra, ACT 0200, Australia \\ ${ }^{2}$ CUDOS, Department of Mathematical Sciences, University of Technology, Sydney, NSW 2007, Australia \\ ${ }^{3}$ CUDOS, School of Physics, University of Sydney, Sydney, NSW 2006, Australia \\ *Corresponding author: ans124@rsphysse.anu.edu.au
}

Received August 28, 2009; revised October 20, 2009; accepted October 26, 2009; posted November 5, 2009 (Doc. ID 116394); published December 2, 2009

\begin{abstract}
We demonstrate that the spatial profiles of both propagating and evanescent Bloch modes in a periodic structure can be extracted from a single measurement of an electric field at the specified optical wavelength. We develop a systematic extraction procedure by extending the concepts of high-resolution spectral methods previously developed for temporal data series to take into account the symmetry properties of Bloch modes simultaneously at all spatial locations. We illustrate the application of our method to a photonic crystal waveguide interface and confirm its robustness in the presence of noise. () 2009 Optical Society of America OCIS codes: $050.5298,230.7370,250.5300$.
\end{abstract}

Periodically modulated optical waveguides offer new possibilities for controlling the propagation of light. Resonant scattering from periodic modulations can be used to tailor the dispersion, enabling in particular a dramatic modification of the group velocity and realization of slow-light propagation. Such fundamental effects can be directly visualized in experiment with near-field measurements, which can be used to recover the amplitude, phase, and polarization of the electric field at all spatial locations in the plane of the waveguide [1]. In this Letter, we demonstrate how this information can be used most efficiently to determine the dispersion characteristics of the guided modes. Our method can also be useful for processing data of numerical simulations.

A commonly used approach to the dispersion extraction is through the spatial Fourier transform (SFT) of the field profiles, since peaks in the Fourier spectra correspond to the wavenumbers of guided modes [2-4]. However, there exists a fundamental limitation on results obtained with SFT: $\Delta k \geqslant 2 \pi / L$, where $\Delta k$ is the resolution of the wavenumber and $L$ is the structure's length. Therefore accurate dispersion results can be obtained only for long waveguides, extending over many periods of the underlying photonic structure. Another limitation of the SFT method is that it cannot provide information on the dispersion of evanescent waves, which may play an important role close to the structure boundaries or interfaces between different waveguides [5]. Moreover, all waves have decaying amplitudes in lossy media such as metal-dielectric metamaterial and plasmonic structures.

Alternative methods for dispersion extraction have been developed to overcome the shortcomings of the SFT method. An interference pattern of two counterpropagating modes in photonic crystals was used to extract their wavenumbers [6]. It was shown that in metamaterials, the effective refractive index can be determined through the extracted phase velocity of a single propagating or evanescent wave $[7,8]$. However, these techniques do not apply in the presence of multiple propagating or evanescent modes.

Recently, it was demonstrated that dispersion extraction in multimode waveguides with in principle unbounded resolution is possible even for short waveguide sections $[9,10]$, using approaches based on an adaptation of high-resolution spectral methods previously developed for the analysis of temporal dynamics $[11,12]$. In this Letter, we introduce an important generalization of such methods, taking into account the spatial symmetry properties of modes in periodic waveguides. We show that beyond the dispersion relations, it is possible to extract the spatial profiles of all guided modes. Our method is applicable to an arbitrary combination of propagating and evanescent waves. We illustrate the application of this general approach by analyzing light dynamics at an interface between photonic crystal waveguides designed for coupling into a slow-light mode [5].

Let us consider a periodic waveguide section, where the light propagation in a particular frequency range is primarily determined by a finite total number of guided modes $(M)$. The value of $M$ can be established based on numerical modeling, taking into account both propagating and evanescent waves. Since each of the modes of a periodic waveguide satisfies the Bloch theorem [13], the complex electric field envelope of a waveguide mode with the index $m$ at the frequency $\omega$ can be expressed as $\psi_{m}(\mathbf{r} ; \omega) \exp \left(i k_{m} z / d\right)$. Here $k_{m}$ are the complex Bloch wavenumbers; $\mathbf{r}=(x, y, z)$, where $x$ and $y$ are the orthogonal directions transverse to the waveguide and $z$ is the direction of periodicity; $d$ is the waveguide period; and $\psi_{m}$ are the periodic Bloch-wave envelope functions: $\psi_{m}(z)=\psi_{m}(z+d)$. Then, the total field inside the waveguide can be presented as a linear superposition of $M$ guided modes with amplitudes $a_{m}$, 


$$
E(\mathbf{r} ; \omega)=\sum_{m=1}^{M} a_{m} \psi_{m}(\mathbf{r} ; \omega) \exp \left(i k_{m} z / d\right)+w(\mathbf{r} ; \omega)
$$

Here $w(\mathbf{r} ; \omega)$ can account for the radiation field due to the excitation of nonguided waves and for vanishingly small evanescent waves that are excluded from consideration, and this term can also appear owing to noise in experimental measurements.

We now present the procedure for the simultaneous extraction of the wavenumbers and profiles of the guided modes. Let us separate the spatial domain in a number of unit cells, $(x, y, z+n d-d)$. We assume here that $z$ belongs to a single unit cell $\left(z_{\min }<z\right.$ $\left.<z_{\min }+d\right)$, and $n=1: N$, where $N$ is the number of cells in the waveguide section. Let us denote with $U_{n}(\mathbf{r} ; \omega)=E(x, y, z+n d-d ; \omega), \quad A_{m}(\mathbf{r} ; \omega)=a_{m} \psi_{m}(\mathbf{r} ; \omega)$ $\times \exp \left(i k_{m} z / d\right)$, and $w_{n}(\mathbf{r} ; \omega)=w(x, y, z+n d-d ; \omega)$. Then, taking into account the periodicity of Blochwave envelopes, Eq. (1) can be written as

$$
U_{n}(\mathbf{r} ; \omega)=\sum_{m=1}^{M} A_{m}(\mathbf{r} ; \omega) \exp \left[i k_{m}(n-1)\right]+w_{n}(\mathbf{r} ; \omega),
$$

where $\mathbf{r}$ belongs to the first unit cell. If one considers this relation for only a single point $\mathbf{r}$ in the unit cell, it becomes mathematically equivalent to the problems considered in spectral analysis of temporal series [11,12], and high-resolution spectral methods can be used to extract the mode wavenumbers [10]. However, the special property of periodic waveguides is that Eq. (2) shall be satisfied simultaneously for all spatial locations $\mathbf{r}$ in the unit cell. This allows us to determine the values of $k_{m}$ and $A_{m}(\mathbf{r} ; \omega)$, provided that the number of measurements exceeds the number of unknowns: $N \times N_{p} \geqslant M \times N_{p}+M_{k}$, where $N_{p}$ is the number of measurement points per unit cell and $M_{k}$ is the number of independent wavenumber values. Then we seek the parameter values that describe most accurately the entire measured field and apply the least-squares method to find a minimum of the functional $W=\Sigma_{\mathbf{r}} \Sigma_{n=1}^{N}\left|w_{n}\right|^{2} / \Sigma_{\mathbf{r}} \Sigma_{n=1}^{N}\left|U_{n}\right|^{2}$, where summation $\Sigma_{\mathbf{r}}$ is performed over all sampling points in one unit cell. For given wavenumbers, the minimum $W_{A}\left(\left\{k_{m}\right\}\right)=\min _{A} W$ is achieved when $\partial W / \partial A_{m}$ $=\partial W / \partial A_{m}^{*}=0$. It follows that for each point $\mathbf{r}$ in a unit cell, the optimal amplitudes satisfy the linear matrix equation $C^{H} \cdot C \cdot \tilde{A}(\mathbf{r} ; \omega)=C^{H} \cdot \tilde{U}(\mathbf{r} ; \omega)$, where components of vector $\widetilde{A}(\mathbf{r} ; \omega)$ are the optimal amplitude values, components of the matrix $C$ are $C_{n p}=\exp \left[i k_{p}(n\right.$ $-1)]$, and vector $\tilde{U}(\mathbf{r} ; \omega)$ components are $U_{n}(\mathbf{r} ; \omega)$ for $p=1: M$ and $n=1: N$. We can show that $W_{A}\left(\left\{k_{m}\right\}\right)$ $=W_{A=\tilde{A}}=1-\Sigma_{\mathbf{r}} \tilde{U}^{H}(\mathbf{r} ; \omega) \cdot C \cdot \widetilde{A}(\mathbf{r} ; \omega) / \Sigma_{\mathbf{r}} \tilde{U}^{H}(\mathbf{r} ; \omega) \cdot \tilde{U}(\mathbf{r} ; \omega)$. The remaining task is to find the absolute minimum $W_{\text {min }}=\min _{\left\{k_{m}\right\}} W_{A}$ (by definition, $W_{A}$ is real and positive). This can be done numerically, for example by using the FMINSEARCH function in MATLAB.

We apply our method to the two-dimensional $[\mathbf{r}$ $=(x, z)]$ photonic crystal waveguide shown in Fig. 1(a), where we indicate the unit cell with shading and illustrate the definition of $U_{n}$. Photonic crystals

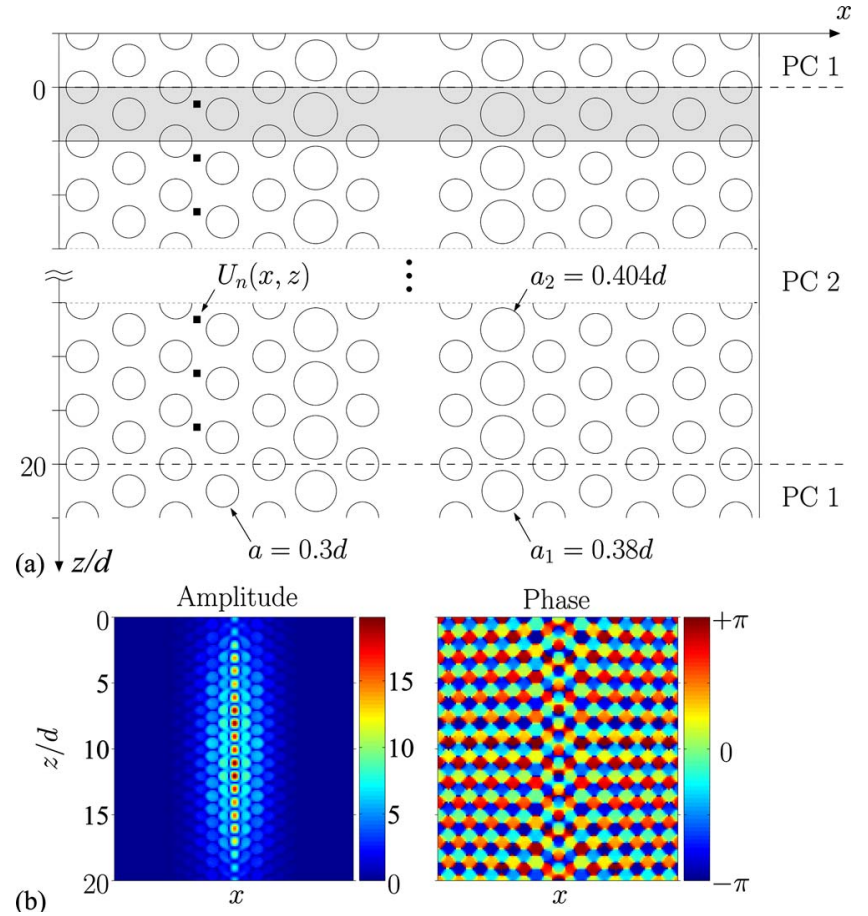

Fig. 1. (Color online) (a) Schematic of a two-dimensional photonic crystal waveguide. The Bloch-wave extraction is performed in the PC2 section. (b) Numerically calculated amplitude (left) and phase (right) of a complex electric field profile at the normalized frequency $d / \lambda \simeq 0.2662$.

are viewed as effective media for generating slow light, because their dispersion characteristics can be controlled with design parameters. However, coupling light into a slow-light waveguide can be a key practical challenge owing to the field mismatch between the incoming mode with high group velocity $v_{g}$ and the slow mode. The photonic crystal waveguide being analyzed here mediates efficient coupling into and out of a slow mode without any matching region, where an evanescent mode (PC2) helps match the fields of the incoming mode (PC1) and the slow mode (PC2) without carrying any energy itself [5]. We aim to extract the individual modes of PC2 from a numerical data of $E(x, z ; \omega)$, such as shown in Fig. 1(b). These electric field profiles were calculated using a Bloch mode scattering matrix approach [14,15], and for our extraction procedure we used the sampling resolution of eight points per period in both $x$ and $z$ directions. In $\mathrm{PC} 2$, the dispersion relation contains an inflection point and therefore the total number of modes that primarily define the light dynamics is $M=6$, accounting for the forward and backward slowlight $(m=1,2)$ and evanescent modes $(m=3,4,5,6)$. The fact that the waveguide has multiple modes and a relatively short length provides a good testing environment for our method.

The spatial spectral analysis can benefit through the application of additional constraints owing to the symmetry of modes in lossless dielectric waveguides, wavenumbers of which are related as $k_{2}=-k_{1}, k_{4}=$ $-k_{3}, k_{5}=k_{3}^{*}$, and $k_{6}=-k_{5}=-k_{3}^{*}$. Therefore $k_{1}$ and $k_{3}$ are the independent parameters that fully define the mode dispersion $\left(M_{k}=2\right)$, and we take this into account for numerical minimization of the func- 


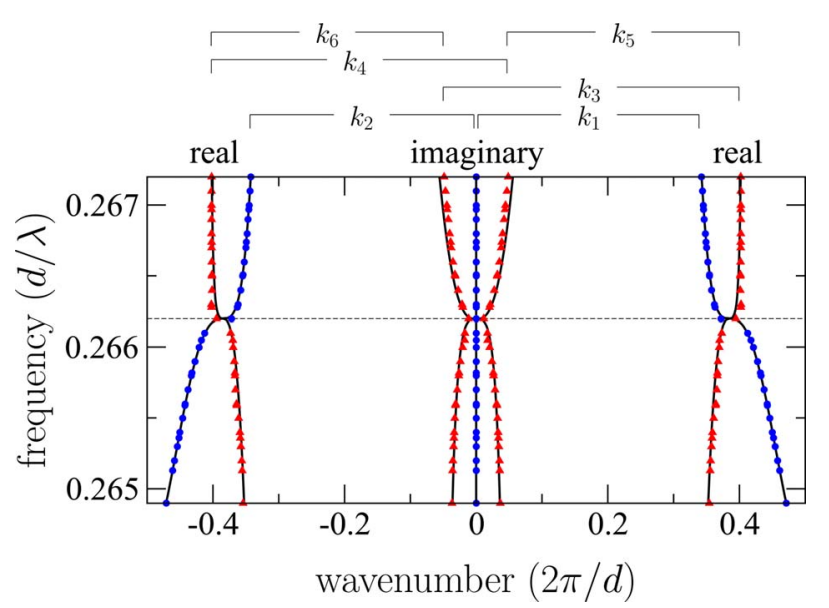

Fig. 2. (Color online) Complex wavenumbers $k_{m}$ extracted with spatial spectral analysis. Propagating and evanescent modes are marked by circles and triangles, respectively. Solid curves show exact dispersion obtained with scattering matrix approach [14,15]. Real and imaginary parts of each mode are indicated above. Horizontal lines at $d / \lambda$ $\simeq 0.2662$ mark the frequency at the inflection point.

tional $W$. We note that under the presence of losses, the wavenumbers would no longer form pairs of complex conjugate numbers. The appearance of such spectral asymmetry could then be used to distinguish between the evanescent mode decay and the amplitude decrease owing to losses.

The extracted wavenumbers $k_{m}$ are shown in Fig. 2 , and they are in good agreement with exact dispersion curves obtained with the scattering-matrix approach [14,15]. Most importantly, we simultaneously extract the profiles of the corresponding Bloch waves, which amplitudes reflect the excitation dynamics. The mode profiles $A_{m}(x, z ; \omega)$ at the slow-light frequency $d / \lambda \simeq 0.2662$ are presented in Fig. 3 . The profiles clearly show the propagating $(m=1,2)$ and evanescent $(m=3,4,5,6)$ modes. This provides an essential insight into the light dynamics, which cannot be inferred directly from the field profiles as shown in Fig. 1(b), or from their SFT spectra.

To test our method in a possible experimental situation where noise is present, we add a normal distribution of random complex numbers to the electric field $U_{n}(x, z)$ with a mean of zero and a standard deviation of 0.05 . We have also considered the effect of a random $1 \%$ perturbation to the radius of the cylinders. We have successfully recovered the mode profiles in the slow-light dispersion region under the effect of such perturbations.

In conclusion, we have presented a general approach for the simultaneous extraction of wavenumbers and amplitude profiles of Bloch waves in periodic waveguides using near-field measurement data or numerical simulation results. We have demonstrated the application of this method for characterization of multiple propagating and evanescent modes, and its robustness under the effect of noise. This approach may provide essential insight into the light dynamics in complex photonic crystal circuits.

\section{References}

1. R. J. P. Engelen, Y. Sugimoto, H. Gersen, N. Ikeda, K.
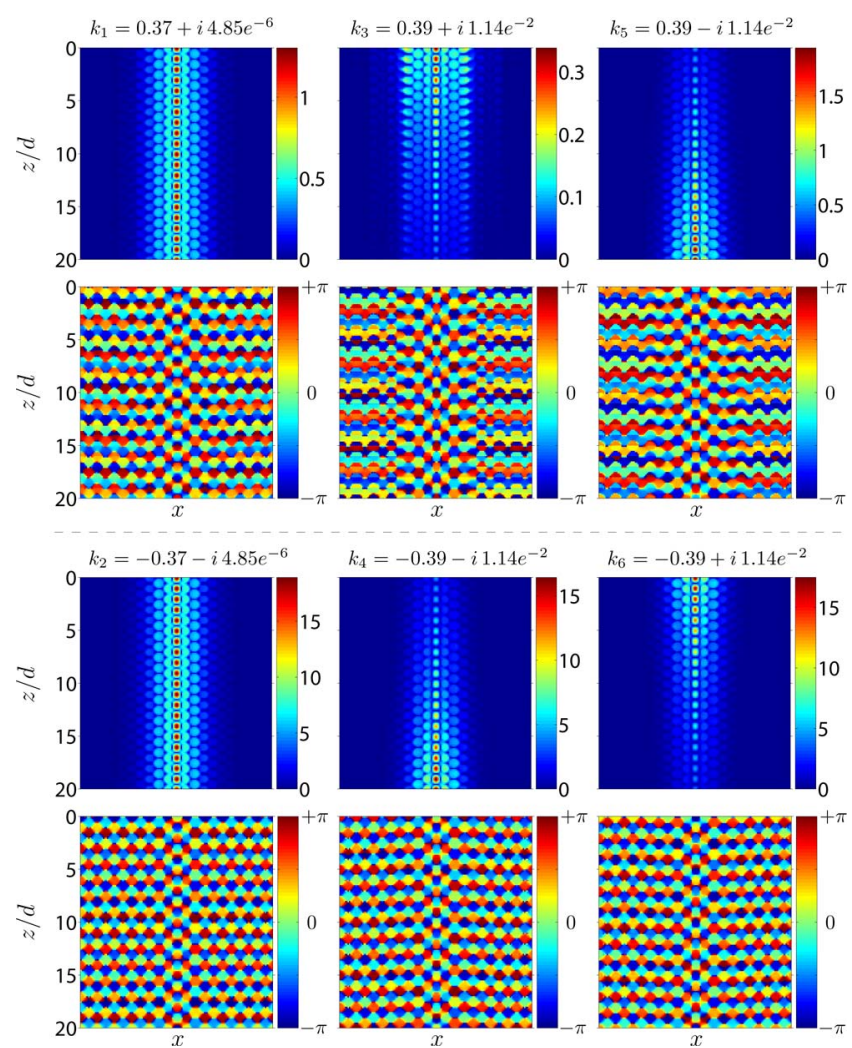

Fig. 3. (Color online) Extracted magnitudes and phases of mode profiles $A_{m}(x, z ; \omega)$ at the slow-light frequency based on the original electric field data shown in Fig. 1(b).

Asakawa, and L. Kuipers, Nat. Phys. 3, 401 (2007).

2. H. Gersen, T. J. Karle, R. J. P. Engelen, W. Bogaerts, J. P. Korterik, N. F. van Hulst, T. F. Krauss, and L. Kuipers, Phys. Rev. Lett. 94, 123901 (2005).

3. H. Gersen, T. J. Karle, R. J. P. Engelen, W. Bogaerts, J. P. Korterik, N. F. van Hulst, T. F. Krauss, and L. Kuipers, Phys. Rev. Lett. 94, 073903 (2005).

4. N. Le Thomas, V. Zabelin, R. Houdre, M. V. Kotlyar, and T. F. Krauss, Phys. Rev. B 78, 125301 (2008).

5. T. P. White, L. C. Botten, C. M. de Sterke, K. B. Dossou, and R. C. McPhedran, Opt. Lett. 33, 2644 (2008).

6. S. H. Fan, I. Appelbaum, and J. D. Joannopoulos, Appl. Phys. Lett. 75, 3461 (1999).

7. B. I. Popa and S. A. Cummer, Phys. Rev. B 72, 165102 (2005).

8. A. Andryieuski, R. Malureanu, and A. V. Lavrinenko, in Theoretical and Computational Nanophotonics, D. Chigrin, ed., AIP Conference Proceedings (AIP, New York, 2009), pp. 37-39.

9. B. Dastmalchi, A. Mohtashami, K. Hingerl, and J. Zarbakhsh, Opt. Lett. 32, 2915 (2007).

10. A. A. Sukhorukov, S. Ha, I. V. Shadrivov, D. A. Powell, and Yu. S. Kivshar, Opt. Express 17, 3716 (2009).

11. R. Roy, B. G. Sumpter, G. A. Pfeffer, S. K. Gray, and D. W. Noid, Phys. Rep. 205, 109 (1991).

12. V. A. Mandelshtam, Prog. Nucl. Magn. Reson. Spectrosc. 38, 159 (2001).

13. J. D. Joannopoulos, R. D. Meade, and J. N. Winn, Photonic Crystals: Molding the Flow of Light (Princeton U. Press, 1995).

14. L. C. Botten, T. P. White, A. A. Asatryan, T. N. Langtry, C. M. de Sterke, and R. C. McPhedran, Phys. Rev. E 70, 056606 (2004).

15. K. Dossou, M. A. Byrne, and L. C. Botten, J. Comput. Phys. 219, 120 (2006). 